Regards sur l'économie allemande

Bulletin économique du CIRAC

$66 \mid 2004$

Varia

\title{
Enseignement supérieur : réforme financière douloureuse
}

Marie-Hélène Pautrat et Werner Zettelmeier

\section{OpenEdition}

Édition électronique

URL : http://journals.openedition.org/rea/3852

DOI : $10.4000 /$ rea.3852

ISBN : 978-2-8218-0828-7

ISSN : 1965-0787

\section{Éditeur}

CIRAC

Édition imprimée

Date de publication : 1 mai 2004

Pagination : 15-18

ISSN : 1156-8992

Référence électronique

Marie-Hélène Pautrat et Werner Zettelmeier, « Enseignement supérieur : réforme financière douloureuse », Regards sur l'économie allemande [En ligne], 66 | mai 2004, mis en ligne le 12 octobre 2009, consulté le 10 décembre 2020. URL : http://journals.openedition.org/rea/3852 ; DOI : https:// doi.org/10.4000/rea.3852 


\section{Enseignement supérieur : réforme financière douloureuse}

\author{
Marie-Hélène Pautrat, Werner Zettelmeier
}

A la veille de la rentrée 2003, la prestigieuse université Humboldt de Berlin créait la surprise en annonçant que la dégradation de sa situation financière ne lui permettait pas d'accepter de nouveaux étudiants. Même si les établissements d'enseignement supérieur allemands, selon leur type et leur profil, ne subissent pas dans la même mesure les répercussions des politiques budgétaires restrictives de leurs tutelles publiques, ils s'accordent généralement pour tirer la sonnette d'alarme : dans un contexte de forte augmentation des effectifs étudiants, l'écart se creuse entre les ressources allouées par les tutelles publiques et les besoins de financement. La question que posent les établissements est dès lors de savoir si le système, dans son état actuel, s'avère capable d'absorber, à qualité constante, une nouvelle demande sociale (et politique) d'enseignement.

A la rentrée universitaire 2003/2004, les établissements allemands d'enseignement supérieur ont enregistré le nombre record de 2,026 millions étudiants inscrits. Les effectifs repartent ainsi à la hausse après un tassement des inscriptions entre 1994 et 1997. Le chiffre record de 2003 peut prêter lieu à deux types d'interprétation: l'une évidemment positive, puisqu'elle témoigne d'une forte propension à étudier et prédit une élévation du niveau général d'éducation. En effet, les données de l'OCDE continuent de souligner la faiblesse relative, en Allemagne, du taux d'inscrits dans le secteur tertiaire A (c'est-à-dire l'ensemble des établissements d'enseignement supérieur à l'exception des écoles supérieures d'administration, les Verwaltungshochschulen) : avec 32,4\% en 2001, il demeure largement en deçà de la moyenne OCDE (46,8 \% ; France : 36,5\%). II est vrai que la formation duale en Allemagne draine une part importante des jeunes d'une classe d'âge et les conduit à des niveaux de qualification technique élevés. Mais la Conférence des ministres de l'éducation des Länder (KMK) s'attend à une hausse soutenue des premières inscriptions dans l'enseignement supérieur, au moins jusqu'en 2008.

Une autre lecture de ces chiffres permet toutefois de mieux appréhender les difficultés actuelles du système d'enseignement supérieur : en 2001, le nombre de places d'études officiellement disponibles dans les établissements s'élevait à 1080 000. A l'augmentation du nombre de nouveaux inscrits, c'est-à-dire à la poursuite de la démocratisation de l'enseignement supérieur, s'ajoute la question spécifique à l'Allemagne d'une durée jugée trop longue des études, et d'un taux d'échec relativement élevé. Le système d'enseignement supérieur et les 373 établissements qui le composent cherchent donc toujours à résoudre la délicate équation : comment absorber les flux d'étudiants tout en tentant d'améliorer les capacités d'accueil et en garantissant un haut niveau de qualité des formations proposées, dans le cadre de l'organisation actuelle du système d'enseignement supérieur. Aujourd'hui pourtant, les établissements allemands estiment que leur situation de sous-financement ne leur permet pas de remplir leurs missions dans des conditions satisfaisantes et tirent le signal d'alarme.

Selon les données de l'OCDE, les autorités publiques allemandes financent à hauteur de $91,8 \%$ le secteur tertiaire de l'éducation en Allemagne, pour une moyenne de $78,6 \%$ dans les pays de l'OCDE (85,7\% en France). Du fait de
Les établissements face à un nouvel accroissement des effectifs

Une équation difficile pour les établissements

Le financement des établissements incombe aux Länder 
Des dépenses en hausse...

... que le financement public couvre de moins en moins

l'organisation fédérale de la RFA, ce sont les Länder, de par leur compétence quasi exclusive pour les questions d'éducation, qui prennent en charge l'essentiel des dépenses publiques d'enseignement supérieur, et en particulier l'ensemble des dépenses de personnel, enseignant ou non. Ainsi, si on s'en tient à l'activité d'enseignement et de recherche des établissements (en excluant l'activité de soins des CHU, les Länder ont couvert $80,2 \%$ des besoins de financement des établissements en 2001, voire près de $90 \%$ pour le seul domaine de l'enseignement : soit une dépense de 16,2 milliards $€$ contre 2,4 milliards $€$ pris en charge par le Bund. La situation des établissements est donc intimement liée aux arbitrages politiques et budgétaires des Länder.

Dès lors, le volontarisme qu'affiche volontiers le gouvernement fédéral en matière d'enseignement ne joue finalement qu'un rôle marginal : son poids dans le financement de l'enseignement supérieur se limite à une participation aux investissements dans le patrimoine immobilier des établissements et, de manière plus indirecte, quoique essentielle, au financement des institutions de recherche universitaire et extra-universitaire (DFG, instituts Max-Planck, Société Fraunhofer, etc.). Si le soutien aux institutions augmente dans le budget 2004, la participation aux programmes de constructions universitaires régresse $(-12,7 \%)$ pour s'établir à 925 millions $€$, retrouvant quasiment son niveau de 1998.

Selon les calculs de l'Office fédéral des Statistiques, les dépenses des établissements se sont élevées en 2001 à 28,7 milliards $€$ (+4 \% environ sur un an), soit 1,4\% du PIB allemand (1,3\% en 1992). Ces chiffres incluent les dépenses des $\mathrm{CHU}$ : ces derniers constituent un facteur de coût essentiel du système universitaire, mais couvrent dans le même temps près de $30 \%$ de leurs dépenses par les recettes de leur activité de soins. Si on inclut les dépenses supplémentaires à la charge des établissements au titre des pensions de retraite et de l'assurance maladie des fonctionnaires (elles incombent à l'employeur public, sur son budget, les fonctionnaires n'étant pas assujettis au régime général de l'assurance sociale) et des bourses pour doctorants et post-docs, le total se monte à 29,8 milliards $€$. Une part prépondérante de ce montant est consacrée aux dépenses courantes des établissements (personnel : 17,5 milliards $€$; fonctionnement : 7,8 milliards $€$ ), les investissements ne s'élevant qu'à 3,2 milliards $€$.

Or dans un contexte économique difficile, les Länder répercutent directement sur les établissements les contraintes budgétaires, et la dotation de base accordée par les tutelles décroît. Une étude du Wissenschaftsrat (2001), organe consultatif chargé d'élaborer des recommandations sur le développement structurel et les investissements dans l'enseignement supérieur et la recherche, avait déjà mis en évidence le décrochage entre la progression du financement de base accordé par les Länder aux universités et le rythme d'évolution des dépenses sur la période 1993-1998, entraînant mécaniquement un recul de ce qui constitue leur socle de financement. Et effectivement, selon l'Office fédéral des Statistiques, la dotation de base ne constituait plus en 2001 que 57,9\% du financement total des établissements (contre $61,6 \%$ en 1993). Mais ce chiffre global dissimule une réalité très disparate et touche inégalement les établissements selon leur type et leur profil.

Sources de financement des établissements du supérieur en \% (2001)

\begin{tabular}{|c|c|c|c|c|c|}
\hline & Universités $^{\star}$ ) & CHU & Beaux-Arts & Fachhochschulen & Ensemble éts. \\
\hline Dotation de base & 79,1 & 30,2 & 96,8 & 90,9 & 57,9 \\
\hline $\begin{array}{c}\text { Recettes } \\
\text { administratives }\end{array}$ & 3,2 & 63,9 & 1,3 & 4,1 & 31,3 \\
\hline Ressources propres & 17,8 & 5,9 & 1,9 & 5 & 10,7 \\
\hline
\end{tabular}

Source: Statistisches Bundesamt 2003, Bericht zur finanziellen Lage des Hochschulen, p. 69; *) sans CHU.

Les établissements doivent renforcer leurs ressources propres
De ce fait, le poids des deux autres catégories de recettes des établissements : les recettes administratives et les ressources propres sur projet (Drittmittel), 
tend à se renforcer dans leur structure de financement. En 2001, les recettes des établissements s'élevaient en effet à 12,1 milliards (+4,9\% sur un an), dont 9 milliards $€$ de recettes administratives et 3,1 milliards $€$ de ressources propres sur projets. Les recettes administratives sont constituées pour l'essentiel par la rémunération des soins hospitaliers, les autres types de recettes (publications, prestations vendues, etc.) restant encore globalement peu significatives en volume. Le développement des Drittmittel poursuit pourtant sa progression depuis 20 ans, mais n'atteignait en 2001 que 10,7\% du financement global des établissements $(5,8 \%$ en 1980$)$. Ces fonds publics ou privés servent à près de $90 \%$ (Office des statistiques), au financement de la recherche universitaire par projets. Une analyse plus fine met dès lors en évidence une forte variation de ce ratio en fonction du type d'établissement et des disciplines enseignées. II est ainsi supérieur dans le cas des universités sans $\mathrm{CHU}$ (près de $18 \%$ ). Mais les établissements dont l'activité de recherche est réduite ne peuvent financer dans la même mesure leur activité par des Drittmittel (5\% en moyenne seulement). C'est le cas pour les Fachhochschulen, ces établissements créés en 1970 et qui dispensent, dans un nombre limité de champs disciplinaires, un enseignement plus court et professionnalisé que les universités. L'indicateur des Drittmittel, bien qu'imparfait, revêt néanmoins une importance croissante dans un contexte ou certains Länder optent pour un financement des établissements en fonction de critères, comme le ratio ressources propres/postes de professeurs.

Financement sur projet des établissements d'enseignement supérieur (Drittmittel) - origine et volume en millions d'euros (2001) -

\begin{tabular}{|c|c|c|c|c|c|}
\hline DFG*$\left.^{*}\right)$ & entreprises & Bund & fondations & org. internationaux & autres secteur public \\
\hline 943 & 831,7 & 735,4 & 206,9 & 193,7 & 165 \\
\hline
\end{tabular}

Source : Office fédéral de la Statistique, Bericht zur finanziellen Lage der Hochschulen, 2003 ; *) DFG : Deutsche Forschungsgesellschaft. Cet organisme soutient financièrement la recherche dans les établissements de l'enseignement supérieur et les centres de recherche extra-universitaires, en distribuant des crédits accordés par le Bund et les Länder.

Dans ce contexte de raréfaction des financements publics, les établissements sont encouragés à diversifier leurs ressources financières. Une enquête menée par l'institut de recherche iw de la Fédération de l'industrie BDI sur la nature de l'activité économique des universités a révélé que, en 2001, une soixantaine d'universités et de Fachhochschulen avait participé à la création de sociétés, et y détenait des participations. Mais le développement d'une activité économique (externalisation, filialisation d'activités commerciales, fundraising, sponsoring, etc.) peut se heurter à des obstacles d'ordre juridique, institutionnel ou statutaire, qui appellent la mise en œuvre de montages complexes (création de filiales, de fondations, etc.). L'exemple de l'activité de formation continue est en cela significatif : alors qu'elle est inscrite dans la loi-cadre sur l'enseignement supérieur, elle reste très timide dans les universités allemandes. En effet, le statut des enseignants du supérieur se révèle peu incitatif à cet égard, car il ne leur permet pas d'exercer cette activité au sein de leur établissement, bien qu'ils puissent la pratiquer librement à l'extérieur. Et le cas échéant, les établissements peuvent être contraints de reverser à leur tutelle une partie des recettes ainsi générées ! Enfin, dernier objet de débat : la participation des étudiants au financement des établissements. Cette question ressurgit régulièrement en Allemagne et n'a pas été réglée définitivement en dépit d'un amendement de la Loi-cadre en août 2002 interdisant le prélèvement de droits de scolarité dans un premier cursus : celui-ci fait aujourd'hui l'objet d'une plainte de certains Länder chrétiens-démocrates devant le Tribunal constitutionnel fédéral, au motif d'une ingérence du Bund non compatible avec la Constitution.

Face à la difficulté de diversifier et d'augmenter de manière conséquente leurs ressources financières, les établissements sont instamment invités par leur tutelle à réorganiser leur mode de gestion budgétaire interne afin d'accroître leurs ... et meilleure efficacité de la gestion du système

Une double contrainte : diversification des ressources... marges de manœuvre financière. Depuis la présentation, en 1994, de « Onze 
thèses pour un renforcement de l'autonomie financière des établissements d'enseignement supérieur » par la Conférence permanente des ministres du Culte et de l'Enseignement des Länder (KMK), de nombreuses pistes ont été expérimentées pour finalement se généraliser sous des formes diverses, fédéralisme oblige, en fonction des Länder : flexibilisation et globalisation des dotations, renforcement de l'autonomie de gestion des établissements, octroi des dotations sur critères et mise en compétition des établissements d'un même Land. Au niveau des établissements, le recours à de nouveaux outils de gestion et procédures budgétaires est donc encouragé : le renouvellement des méthodes comptables a pour fonction de mieux appréhender la réalité de la situation financière et budgétaire des établissements et d'ériger des clés différenciées de répartition des ressources. Mais l'utilisation de ces outils comptables, si elle apporte une transparence salutaire dans la gestion des établissements, n'est bien sûr pas sans susciter des craintes légitimes car elle met implicitement en évidence des arbitrages politiques. Or, certaines missions de l'université, comme la transmission des savoirs ou la recherche fondamentale, ne peuvent être pleinement appréhendées sous l'angle exclusif de l'identification des coûts. Mais les établissements ont-ils seulement le choix face à une triple pression des tutelles, des financements européens sur projet qui exigent une identification exacte des coûts, et également une opinion publique qui revendique aujourd'hui une plus grande rationalisation de la dépense publique?

DEPUIS PRÈS DE DIX ANS, LES ÉTABLISSEMENTS allemands vivent une réforme silencieuse, mais douloureuse, de leur structure de financement. Les Länder qui jouent un rôle prépondérant dans le financement de l'enseignement supérieur ont multiplié les expérimentations mais se rejoignent tous pour accorder, du moins sur le papier, une plus grande autonomie de gestion. En contrepartie, les établissements sont instamment invités à modifier et à optimiser leur mode de gestion financière et budgétaire interne : financement sur critères, budgets globaux, comptabilité analytique sont autant d'outils qui ont déferlé, bon an, mal an, en moins de 10 ans sur les universités allemandes, archétypes de la gestion camérale. Mais à budget au mieux constant et le plus souvent déclinant, les établissements alertent aujourd'hui les pouvoirs publics : les ambitions proclamées pour la société de la connaissance se marient mal avec la réalité d'organismes qui peinent à assurer aujourd'hui leur gestion quotidienne.

\section{Indications bibliographiques}

CIRAC, Modernisation et innovation dans les universités en France et en Allemagne, étude réalisée avec le concours de la Fondation Bosch et de la CDC (à paraître) DESTATIS, Bericht zur finanziellen Lage der Hochschulen, Wiesbaden, 2003

Konegen-GrenieR Ch., Winde A. M., Hochschulunternehmen. Ergebnisse einer empirischen Befragung, Beiträge des IW Köln zur Gesellschafts- und Bildungspolitik Nr. 255, Cologne, 2002

KULtUSministerKonferenz, 11 Thesen zur Stärkung der Finanzautonomie der Hochschulen und Materialband, Bonn, 1994; et Prognose der Studienanfänger, Studierenden und Hochschulabsolventen bis 2020, Statistische Veröffentlichungen. Dokumentation Nr. 167, mars 2003

LASSERRE R., "L'exemple allemand: une modernisation sous contrainte ", in Dossier : "L'enseignement supérieur dans la compétition mondiale ", Sociétal, $\mathrm{n}^{\circ} 44$, second trimestre 2004

WISSENSCHAFTSRAT, Drittmittel und Grundmittel der Hochschulen 1993-1998, Cologne, 2000 